\title{
Estudio inicial de las propiedades psicométricas de las escalas motivacionales del PALS (Patterns of Adaptive Learning Scales) centradas en el alumno
}

\author{
Initial study of the psychometric properties of the motivational scales of the PALS \\ (Patterns of Adaptive Learning Scales) focusing on the student
}

\author{
Fermín Torrano, ${ }^{a}$ María Carmen González-Torres ${ }^{b}$
}

${ }^{\text {a } U n i v e r s i d a d ~ I n t e r n a c i o n a l ~ d e ~ l a ~ R i o j a ~(U N I R), ~ E s p a n ̃ a ~}$

Telf.: (34) 6351791 25. Correo electrónico: fermin.torrano@unir.net

${ }^{\mathrm{b}}$ Facultad de Educación y Psicología, Universidad de Navarra, España

Telf.: (34) 9484256 00. Correo electrónico: mgonzalez@unav.es

\begin{abstract}
RESUMEN
El objetivo de esta investigación es realizar un estudio inicial de las propiedades psicométricas de las escalas motivacionales del Patterns of Adaptive Learning Scales (PALS) centradas en el alumno. La muestra estuvo compuesta por 374 alumnos españoles de $2^{\circ}$ curso de Educación Secundaria Obligatoria. En primer lugar, se estudió el comportamiento psicométrico de cada ítem. En segundo lugar, se obtuvo la estimación de la fiabilidad a través del coeficiente alfa de Cronbach. Finalmente, se utilizó el análisis factorial, con objeto de examinar el grado de ajuste de los datos a la estructura teórica propuesta. Los resultados evidencian, con algunas matizaciones, que nuestra versión posee las propiedades suficientes para poder utilizarse en la práctica educativa y en la investigación motivacional en alumnos de educación secundaria, aunque las dificultades encontradas para diferenciar las dimensiones approach-avoidance de las metas de rendimiento nos aconsejan ser prudentes y recomendar futuros estudios en esta dirección.
\end{abstract}

Palabras clave: motivación, metas académicas, validación de constructo, PALS.

\section{ABSTRACT}

The purpose of this paper is to carry out an initial validation study of the motivational scales of the Patterns of Adaptive Learning Scales (PALS) focusing on the student. The sample was composed of 374 Spanish students from $8^{\circ}$ grade of middle-school. For this task, we focused on different procedures of analysis: we made confirmatory and exploratory factorial analysis. On the other hand, we calculate psychometric indexes, and the internal consistency. The results show that our version has the appropriate characteristics to be used on the educational practice and in the motivational research of secondary education students. However, the difficulties found to differentiate the "approach-avoidance" dimensions from the performance goals make us be prudent and recommend future studies of validation in such direction.

Key words: academic motivation, achievement goal, validation study, PALS. 
Estudios Pedagógicos XLII, $\mathrm{N}^{\circ}$ 3: 391-412, 2016

ESTUDIO INICIAL DE LAS PROPIEDADES PSICOMÉTRICAS DE LAS ESCALAS MOTIVACIONALES DEL PALS

(PATTERNS OF ADAPTIVE LEARNING SCALES) CENTRADAS EN EL ALUMNO

\section{INTRODUCCIÓN}

La amplia investigación que se inició sobre aprendizaje autorregulado en los años 80 del siglo XX muestra, de una manera clara, que para tener éxito en la escuela los estudiantes necesitan tanto habilidades cognitivas (skill) como motivacionales (will) (Torrano y González-Torres, 2004; Zimmerman y Schunk, 2011). Bajo este marco de referencia, en las últimas décadas se ha desplegado un gran número de líneas de investigación orientadas al estudio de las interrelaciones entre los rasgos cognitivos y motivacionales, así como a la puesta en marcha de programas de intervención dirigidos tanto a profesores como a alumnos de primaria, secundaria y universidad (Butler, Schnellert y Cartier, 2013; Daura, 2011; Dignath y Buttner, 2008; González-Cabanach et al., 2007; González-Torres y Torrano, 2013; Rosario et al., 2015; Schunk y Zimmerman, 2007; Zimmerman y Schunk, 2011). Al mismo tiempo, se han ido perfeccionado los métodos de evaluación en este campo (González-Torres y Torrano, 2008; Winne, en prensa; Zimmerman, 2008).

\subsection{LA TEORÍA SOBRE METAS ACADÉMICAS (ACHIEVEMENT GOAL THEORY)}

Dentro de los diferentes constructos motivacionales que ejercen un significativo rol mediador en la implicación en el aprendizaje, y como consecuencia, en el rendimiento, destacan las metas. En este sentido, la teoría sobre metas en contextos escolares (Achievement goal theory) ha sido uno de los principales enfoques de la investigación motivacional, dirigiéndose a estudiar las intenciones o razones por las que los alumnos se comprometen y persisten en su aprendizaje (Anderman y Wolters, 2006; De la Fuente, 2004; Meece, Anderman y Anderman, 2006; Senko, Hulleman y Harackiewicz, 2011; Valle et al., 2009).

Inicialmente, en las primeras teorizaciones, los autores distinguieron dos tipos de metas a las que se orientaban los estudiantes: las metas de aprendizaje (también llamadas metas de dominio o centradas en la tarea — mastery/task goal—), que guían la conducta hacia el desarrollo de nuevas capacidades, conocimientos y al logro de un sentido de dominio basado en estándares autoestablecidos, y las metas de rendimiento o de desempeño (también llamadas metas de habilidad o metas centradas en el yo — performancel ability/ego goal-) que orientan al estudiante primeramente a centrarse en demostrar que posee capacidad y en lograr un sentido de autovalía (muchas veces, superando a otros en rendimiento), obteniendo además un reconocimiento público por ello. La mayor parte de la investigación ha puesto de relieve los beneficios de las metas de aprendizaje. A este respecto, existen muchos estudios que apuntan al hecho de que centrarse en el dominio de la tarea está positivamente asociado con patrones atribucionales adaptativos, actitudes positivas hacia el aprendizaje, uso de estrategias de aprendizaje que favorecen la comprensión profunda, el recuerdo y la autorregulación cognitiva, preferencia por actividades desafiantes, altos niveles de interés, persistencia y búsqueda de ayuda cuando se precisa (Anderman, Urdan y Roeser, 2003; Harackiewicz et al., 2002; Meece et al., 2006).

Respecto a los beneficios de las metas de rendimiento, la lectura todavía está lejos de ser clara. Se ha evidenciado que generan patrones motivacionales menos adaptativos, puesto que los estudiantes están demasiado preocupados por ser los mejores, rendir mejor que otros, o bien, no ser vistos como incompetentes, lo que les genera más afectos negativos, como ansiedad. La preocupación y atención dedicada a cómo uno rinde en 
relación a los otros contribuye a que los estudiantes prefieran tareas menos desafiantes por temor a fracasar y a que abandonen más fácilmente frente a las dificultades; conduce a una mayor distracción de la tarea a la par que a una menor implicación cognitiva en la misma; un mayor uso de estrategias de procesamiento superficial y afectos más negativos hacia la escuela (Anderman et al., 2003; Barron y Harackiewicz, 2003; Harackiewicz et al., 2002; Kaplan y Maehr, 2007; Maehr y Zusho, 2009; Valle et al., 2009). No obstante, los resultados han sido dispares y, en algunas ocasiones, contradictorios, lo que ha ocasionado un amplio debate en la literatura acerca de sus hipotéticos efectos (Senko et al., 2011). El motivo es que en las primeras escalas desarrolladas para medir las metas de rendimiento no se distinguía claramente entre la orientación a mostrar capacidad y la orientación a evitar aparecer incompetente en comparación con otros. Estos componentes, de aproximación y evitación, sin embargo, ya habían sido distinguidos en las teorías tradicionales de motivación de logro, como en el modelo propuesto por Atkinson en 1957 (motivo de éxito y motivo de evitar el fracaso).

En este contexto, algunos autores (Barron y Harackiewicz, 2003; Darnon et al., 2007; Harackiewicz et al., 2002; Midgley, Kaplan y Middleton, 2001) comenzaron a diferenciar estos dos componentes approach-avoidance de las metas de rendimiento, elaborando nuevos modelos multidimensionales (de la Fuente, 2004). De esta manera, las metas de rendimiento (aproximación) se definieron como el deseo de los estudiantes de demostrar competencia y rendir mejor que otros, y las metas de rendimiento (evitación) como el deseo de evitar aparecer incompetentes. Si bien la orientación a metas de rendimiento (en su estilo de evitación) se ha asociado más claramente con un patrón de indefensión, bajas percepciones de autoeficacia, mayor uso de estrategias egodefensivas (self-handicapping) y peor rendimiento (por ejemplo, Maehr y Zusho, 2009; Midgley y Urdan, 2001; Urdan, 2004), todavía existe una amplia controversia acerca de los beneficios de las metas de rendimiento en el caso de su componente de aproximación (Elliot y Moller, 2003; Linnenbrink, 2005; Meece et al., 2006; Smith y Sinclair, 2005; Valle et al., 2009). Por ejemplo, algunos estudios indican que están positivamente asociadas con el rendimiento (especialmente, en estudiantes universitarios), con el autoconcepto académico y con el valor dado a la tarea, y que no necesariamente producen una disminución de la implicación cognitiva y del uso de estrategias de autorregulación (Brophy, 2005; Harackiewicz et al., 2008; Hulleman et al., 2008; Muis y Winner, 2012; Wigfield y Cambria, 2010). Sin embargo, otros autores destacan relaciones neutrales o negativas con esos mismos constructos (Anderman et al., 2003; Harackiewicz et al., 2002).

En síntesis, y a pesar de estas discrepancias, nos gustaría señalar que, actualmente, en los nuevos modelos multidimensionales de metas se está superando la tendencia a considerar que las metas de rendimiento (en su estilo de aproximación) son inadecuadas para promover una motivación óptima y el deseo de aprender. De hecho, como ya señaló Dweck (1986) y otros (De la Fuente, 2004; González-Torres, 1997; Midgley et al., 2001; Linnenbrink y Pintrich, 2003; Valle et al., 2009), los alumnos únicamente interesados en metas de aprendizaje, pero no en alcanzar otros objetivos (sacar buenas notas, hacer las tareas que se mandan en clase, lograr los objetivos de clase), pueden estar actuando contra sus intereses e, incluso, pueden poner en riesgo oportunidades para su futuro académico y profesional (acceso a determinadas carreras universitarias, inserción profesional, etc.).

Por último, la investigación centrada en el estudio de metas desde una perspectiva social ha puesto de relieve que una orientación hacia metas sociales, sobre todo hacia 
aquellas relacionadas con la responsabilidad social, en coordinación con una orientación hacia metas académicas (de aprendizaje y de aproximación al rendimiento), es una de las maneras más viables y beneficiosas de aumentar el aprendizaje y el rendimiento (modelos de múltiples metas) (Patrick, Anderman y Ryan, 2002; Wentzel, 2003).

\subsection{EVALUACIÓN DE LAS METAS ACADÉMICAS}

En las dos últimas décadas, en el campo del aprendizaje autorregulado, se ha construido un gran número de escalas dirigidas a evaluar de una forma objetiva la presencia de distintos atributos motivacionales latentes en los sujetos (p. ej. Winne, en prensa; Zimmerman, 2008). En este contexto, el proceso de validación cobra una vital importancia, ya que los resultados que se obtengan ayudarán a confirmar o rechazar la validez de los mismos y también las teorías de los constructos de referencia. Hasta la fecha, los cuestionarios de autoinforme, a pesar de sus limitaciones, son los procedimientos más utilizados para medir los aspectos cognitivos y motivacionales, debido a su facilidad para el diseño, administración e interpretación de los resultados (González-Torres y Torrano, 2008).

En el caso de las metas, algunos de los cuestionarios más utilizados son: Achievement Goal Questionnaire (Elliot y McGregor, 2001), la escala de Skaalvik (1997), The Learning and Strategies Study Inventory (LASSI) (Weinstein, Schulte y Palmer, 1987), The Motivated Strategies for Learning Questionnaire (MSLQ) (Pintrich et al., 1991), The Components of Self-Regulated Learning (CSRL) (Niemivirta, 1998) y el Patterns of Adaptive Learning Scales (PALS a partir de ahora), empleado en nuestro estudio.

\section{OBJETIVOS E HIPÓTESIS}

El objetivo de la presente investigación es llevar a cabo un estudio inicial de las propiedades psicométricas de las escalas motivacionales del PALS centradas en el alumno, uno de los instrumentos más empleados para evaluar aspectos motivacionales tanto en el ámbito escolar (Putwain, 2013) como en otros campos (Hershkovitz et al., 2013; Hoffman et al., 2014; Madjar, Bachner y Kushnir, 2011). A través de este proceso se pretende comprobar si las medidas que se obtienen son adecuadas y si nuestra versión de dichas escalas muestra una estructura interna relativamente consistente y acorde con la hallada por los autores del instrumento original.

\section{MÉTODO}

\subsection{PARTICIPANTES}

Según los datos proporcionados por el Departamento de Educación del Gobierno de Navarra en el momento de realizarse el estudio, la población de referencia estaba constituida por 2268 estudiantes agrupados en 8 centros públicos y 20 privados (ver Tabla 1). 
Tabla 1. Tamaño de la población y de la muestra por centros públicos y privados

\begin{tabular}{|l|c|c|c|}
\hline & C. PÚBLICOS & C. PRIVADOS & TOTAL \\
\hline Población & 832 & 1436 & 2268 \\
\hline Muestra esperada & 154 & 266 & 420 \\
\hline Muestra obtenida & 131 & 243 & 374 \\
\hline
\end{tabular}

Para la determinación del tamaño de la muestra, asumimos, como error muestral máximo, un valor de 0.22 desviaciones típicas. Debido a que la desviación típica media de los ítems de los cuestionarios es de 1.71 puntos, estaríamos operando con un error absoluto de .38 puntos. Como vemos en la Tabla 1, el número total estimado de alumnos que deberían formar parte de la muestra es de 420, 154 de centros públicos y 266 de centros privados, lo que corresponde aproximadamente al $18 \%$ de la población total.

El total de la muestra conseguida fue de $\mathrm{N}=374$ alumnos (131 de centros públicos y 243 de centros privados, que constituye el 16,5\% de la población de los estudiantes matriculados en $2^{\circ}$ curso de la ESO (edad media de los sujetos: $13-14$ años; $51 \%$ chicos y $40 \%$ chicas; nivel socioeconómico medio-alto) en los centros de educación secundaria de Pamplona y su comarca.

\subsection{INSTRUMENTO}

El PALS es una batería de escalas desarrolladas en los años 90 y refinada a lo largo de la siguiente década por Carol Midgley y su equipo de investigación de la School of Education de la Universidad de Michigan, con objeto de examinar las relaciones entre el ambiente de aprendizaje, la motivación, el afecto y el comportamiento de los estudiantes (Midgley et al., 2000). Es un instrumento que está muy bien fundamentado teóricamente, concretamente, en la teoría de metas vista anteriormente y en las investigaciones de Carol Ames (1992). Este conjunto de escalas se ha aplicado tanto en primaria (Anderman y Anderman, 1999; Anderman y Midgley, 1997) como en secundaria (Kaplan y Midgley, 1997; Midgley y Urdan, 2001).

La batería original se estructura en un conjunto de escalas referidas al alumno y otras centradas en el profesor. Dentro del primer grupo (alumno), nos encontramos con cinco escalas que evalúan las metas académicas que persiguen los estudiantes, sus percepciones acerca de las metas que tienen su profesores, sus percepciones acerca de las metas que se enfatizan en el aula para implicarse en el aprendizaje, sus percepciones del contexto familiar, así como un conjunto de creencias, actitudes y estrategias motivacionales (como las creencias de autoeficacia o las estrategias self-handicapping). Dentro del segundo, dirigidas al profesor, se sitúan tres escalas que estiman las percepciones que tienen estos de las metas de la escuela, sus metas respecto de la instrucción, así como sus creencias de autoeficacia docente.

Una de las principales razones por las que se decidió utilizar este instrumento fue debido a la distinción que establecía entre los componentes de aproximación y evitación de las metas de rendimiento, y entre metas personales y contextuales, en línea con lo 
propuesto por las últimas revisiones de la teoría de metas (Negru y Damian, 2010; Urdan y Schoenfelder, 2006). Asimismo, en muestras de adolescentes y en el caso de las matemáticas, se ha comprobado que el PALS es una de las medidas de orientación de metas más válida y fiable (Patrick, Ryan y Kaplan, 2007; Strobel, 2010).

En nuestro estudio hemos traducido y adaptado al castellano la última versión elaborada en el año 2000. En concreto, se utilizaron las siguientes escalas referidas al alumno (ver Figura 1):

- Orientación a metas académicas (Personal Achievement Goal Orientations): la orientación a metas se refiere a la percepción por parte del estudiante de las razones por las que se implica en el aprendizaje. Esta dimensión engloba tres subescalas: 1) Orientación a metas de aprendizaje (Mastery Goal Orientation), que hace referencia al grado en el que el alumno se implica en el aprendizaje por razones tales como el reto, dominio o desarrollo de capacidades (ítems 4, 9, 11, 13 y 20); 2) Orientación a metas de rendimiento, que a su vez se divide en otras dos, que se refieren al grado en el que el estudiante participa en el aprendizaje por el deseo de demostrar competencia (Performance-Approach Goal Orientation) (ítems 3, 10, 14, 17 y 19) o con el fin de evitar demostrar la propia incompetencia (Performance-Avoid Goal Orientation) (ítems 2, 12, 21 y 23).

- Percepción de las metas del aula (Perception of Classroom Goal Structures): esta dimensión se refiere a las percepciones que tienen los estudiantes acerca de las razones que se enfatizan en el aula para implicarse en el aprendizaje. Al igual que antes, dicha dimensión se desglosa en tres subescalas: 1) Percepción de la clase orientada al dominio (Classroom Mastery Goal Structure), que se refiere a que los alumnos perciben en el aula que una de las razones importantes para implicarse en el trabajo académico es desarrollar la propia competencia (p. ej., aprender nuevas cosas, entender el material (ítems 26, 28, 30, 33, 35 y 37); 2) Percepción de la clase orientada al rendimiento, que se divide a su vez en otras dos, que comprenden las percepciones que desarrollan los alumnos en el aula referidas a que el objetivo de implicarse en el aprendizaje es demostrar competencia (Classroom PerformanceApproach Goal Structure) (ítems 29, 31 y 38) o evitar demostrar la propia incompetencia (Classroom Performance-Avoidance Goal Structure) (ítems 27, 32, 34, 36 y 39).

- Autoeficacia académica: que comprende las percepciones de competencia que desarrollan los estudiantes a la hora de realizar las tareas académicas (ítems 1, 5, 22, 24 y 25).

- $\quad$ Uso de estrategias self-handicapping: que se refiere a las estrategias, excusas, pretextos, que utilizan algunos estudiantes para justificarse ante un mal resultado o un bajo rendimiento académico, y, de esta manera, el fracaso pueda ser atribuido a esas mismas causas y no a la falta de capacidad (ítems 6, 7, 8, 15, 16 y 18). 
Figura 1. Escalas del PALS empleadas

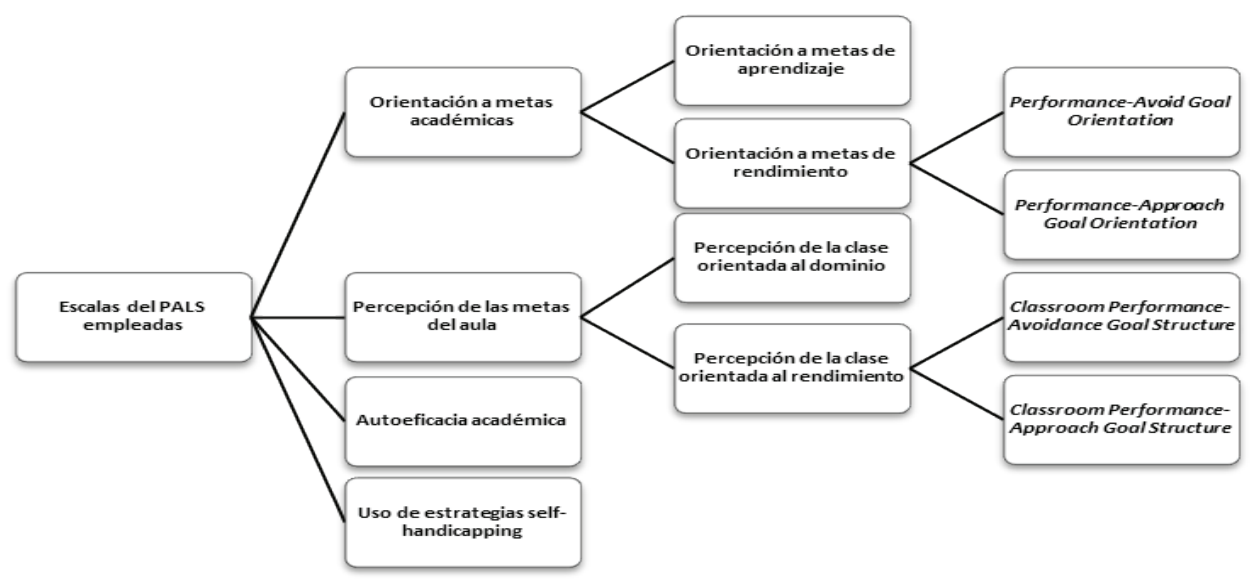

\subsection{PROCEDIMIENTO}

En primer lugar, se tradujeron y adaptaron las escalas del PALS que iban a formar parte del presente estudio, decidiendo aplicarlas en el $2^{\circ}$ curso de Educación Secundaria Obligatoria (ESO), en la asignatura de matemáticas. La razón de centrarnos en esta materia fue debido a nuestro interés por conocer los aspectos afectivos-motivacionales imbricados en el aprendizaje de las matemáticas, en línea con importantes líneas de investigación orientadas al estudio de estos factores y al desarrollo de propuestas de intervención en este ámbito dirigidas tanto a los profesores como a los alumnos (cfr. Blanco, 2012; Sarabia e Iriarte, 2011; Turner et al., 2002).

En el cuestionario original PALS los alumnos deben contestar con arreglo a una escala tipo Likert de 5 puntos ( $1=$ en absoluto cierto; $3=$ en algunas ocasiones cierto; $5=$ muy cierto). Sin embargo, como el estudio es mucho más amplio que lo que aquí presentamos (en concreto, se empleó junto con las escalas del cuestionario Motivated Strategies for Learning Questionnaire o MLSQ en la versión validada por Roces, Tourón y GonzálezTorres, de 1995, cuyo formato de respuesta es de 7 puntos), se creyó conveniente cambiar ligeramente la forma de puntuación de las escalas del PALS, pasando de una escala de 5 puntos a una de 7 (1= no, nunca; $3=$ más bien no, alguna vez; $5=$ más bien sí, con bastante frecuencia; $7=$ sí, siempre).

\subsection{ANÁLISIS DE DATOS}

Para el análisis inicial de la estructura interna de las escalas seleccionadas, nos centramos en tres procedimientos. En primer lugar, para el estudio del comportamiento de cada ítem, calculamos los valores medios, las desviaciones típicas y la correlación de cada ítem con el total de su escala, como índice de homogeneidad. En segundo lugar, se llevó a cabo la estimación de la fiabilidad para cada una de las escalas a través del coeficiente alfa de Cronbach, obteniendo de esta forma un indicador de la consistencia interna (o precisión) de cada una de ellas. Finalmente, después de un primer análisis factorial exploratorio, se 
Estudios Pedagógicos XLII, Nº 3: 391-412, 2016

ESTUDIO INICIAL DE LAS PROPIEDADES PSICOMÉTRICAS DE LAS ESCALAS MOTIVACIONALES DEL PALS (PATTERNS OF ADAPTIVE LEARNING SCALES) CENTRADAS EN EL ALUMNO

realizó un análisis confirmatorio, con objeto de estudiar el grado de ajuste de los datos a la estructura teórica propuesta por los autores de este instrumento. Todos estos análisis se realizaron con el programa SPSS (Norusis, 2011) y con el programa AMOS (Analysis of Moment Structures) (Arbuckle, 2010).

\section{RESULTADOS}

\subsection{ESTUDIO DESCRIPTIVO DE LOS ÍTEMS}

En la Tabla 2 se recogen los estadísticos descriptivos (valores medios y desviaciones típicas) de cada uno de los ítems pertenecientes a cada dimensión motivacional, así como la correlación de cada uno con el total de la escala a la que pertenece, lo que indica el grado de homogeneidad con la misma. Las correlaciones que se presentan están corregidas, esto es, se ha eliminado de la puntuación total la puntuación en el ítem correspondiente.

Tabla 2. Media, desviación típica y correlación ítem-total para cada uno de los ítems motivacionales

\begin{tabular}{|l|c|c|c|}
\hline METAS APRENDIZAJE & MEDIA & SD & CORR. ÍTEM-TOT \\
\hline Ítem 4 & 5.19 & 1.50 & .68 \\
\hline Ítem 9 & 5.43 & 1.55 & .74 \\
\hline Ítem 11 & 5.27 & 1.51 & .80 \\
\hline Ítem 13 & 5.47 & 1.50 & .64 \\
\hline Ítem 20 & 5.58 & 1.43 & .75 \\
\hline Total Escala & 5.39 & 1.50 & \\
\hline
\end{tabular}

\begin{tabular}{|l|c|c|c|}
\hline METAS RENDIMIENTO $($ APPROACH $)$ & MEDIA & SD & CORR. ÍTEM-TOT \\
\hline Ítem 3 & 3.15 & 1.91 & .65 \\
\hline Ítem 10 & 3.11 & 1.73 & .75 \\
\hline Ítem 14 & 2.65 & 1.62 & .70 \\
\hline Ítem 17 & 2.38 & 1.69 & .76 \\
\hline Ítem 19 & 2.43 & 1.67 & .78 \\
\hline Total Escala & 2.74 & 1.72 & \\
\hline
\end{tabular}

\begin{tabular}{|l|c|c|c|}
\hline METAS RENDIMIENTO (AVOIDANCE) & MEDIA & SD & CORR. ÍTEM-TOT \\
\hline Ítem 2 & 4.07 & 2.23 & .56 \\
\hline Ítem 12 & 2.92 & 1.92 & .58 \\
\hline Ítem 21 & 3.85 & 2.10 & .58 \\
\hline Ítem 23 & 3.02 & 1.94 & .65 \\
\hline Total Escala & 3.46 & 2.05 & \\
\hline
\end{tabular}


Estudios Pedagógicos XLII, Nº 3: 391-412, 2016 ESTUDIO INICIAL DE LAS PROPIEDADES PSICOMÉTRICAS DE LAS ESCALAS MOTIVACIONALES DEL PALS (PATTERNS OF ADAPTIVE LEARNING SCALES) CENTRADAS EN EL ALUMNO

\begin{tabular}{|l|c|c|c|}
\hline METAS CONTEXTO APRENDIZAJE & MEDIA & SD & CORR. ÍTEM-TOT \\
\hline Ítem 26 & 6.03 & 1.29 & .55 \\
\hline Ítem 28 & 5.27 & 1.55 & .40 \\
\hline Ítem 30 & 5.73 & 1.48 & .55 \\
\hline Ítem 33 & 6.08 & 1.36 & .46 \\
\hline Ítem 35 & 5.85 & 1.36 & .65 \\
\hline Ítem 37 & 5.76 & 1.57 & .42 \\
\hline Total Escala & 5.79 & 1.43 & \\
\hline
\end{tabular}

\begin{tabular}{|l|c|c|c|}
\hline METAS CONTEXTO RENDIMIENTO $($ APPROACH $)$ & MEDIA & SD & CORR. ÍTEM-TOT \\
\hline Ítem 29 & 4.96 & 1.78 & .61 \\
\hline Ítem 31 & 4.64 & 1.75 & .52 \\
\hline Ítem 38 & 5.10 & 1.68 & .57 \\
\hline Total Escala & 4.90 & 1.74 & \\
\hline
\end{tabular}

\begin{tabular}{|l|c|c|c|}
\hline METAS CONTEXTO RENDIMIENTO (AVOIDANCE) & MEDIA & SD & CORR. ÍTEM-TOT \\
\hline Ítem 27 & 3.02 & 1.86 & .67 \\
\hline Ítem 32 & 3.04 & 1.92 & .67 \\
\hline Ítem 34 & 2.82 & 1.74 & .67 \\
\hline Ítem 36 & 3.10 & 2.05 & .68 \\
\hline Ítem 39 & 2.73 & 1.87 & .67 \\
\hline Total Escala & 2.94 & 1.89 & \\
\hline
\end{tabular}

\begin{tabular}{|l|c|c|c|}
\hline AUTOEFICACIA & MEDIA & SD & CORR. ÍTEM-TOT \\
\hline Ítem 1 & 4.73 & 1.38 & .57 \\
\hline Ítem 5 & 4.59 & 1.72 & .57 \\
\hline Ítem 22 & 5.40 & 1.72 & .61 \\
\hline Ítem 24 & 5.31 & 1.64 & .68 \\
\hline Ítem 25 & 5.10 & 1.80 & .65 \\
\hline Total Escala & 5.03 & 1.65 & \\
\hline
\end{tabular}

\begin{tabular}{|l|c|c|c|}
\hline SELF-HANDICAPPING & MEDIA & SD & CORR. ÍTEM-TOT \\
\hline Ítem 6 & 2.66 & 1.58 & .52 \\
\hline Ítem 7 & 2.18 & 1.50 & .57 \\
\hline Ítem 8 & 1.87 & 1.20 & .51 \\
\hline Ítem 15 & 2 & 1.38 & .54 \\
\hline Ítem 16 & 2.19 & 1.62 & .57 \\
\hline Ítem 18 & 2.34 & 1.56 & .53 \\
\hline Total Escala & 2.65 & 1.76 & \\
\hline
\end{tabular}


Estudios Pedagógicos XLII, Nº 3: 391-412, 2016

ESTUDIO INICIAL DE LAS PROPIEDADES PSICOMÉTRICAS DE LAS ESCALAS MOTIVACIONALES DEL PALS

(PATTERNS OF ADAPTIVE LEARNING SCALES) CENTRADAS EN EL ALUMNO

\subsection{ESTUDIO DE LA FIABILIDAD}

En la Tabla 3 se recogen los valores del coeficiente alfa de Cronbach para cada una de las escalas motivacionales seleccionadas.

Tabla 3. Fiabilidades (alfa de Cronbach) de las escalas de motivación

\begin{tabular}{|l|c|c|}
\hline \multicolumn{1}{|c|}{ ESCALA } & PRESENTE ESTUDIO & PALS \\
\hline Metas aprendizaje & .88 & .85 \\
\hline Metas rendimiento (approach) & .89 & .89 \\
\hline Metas rendimiento (avoidance) & .78 & .74 \\
\hline Metas contexto aprendizaje & .76 & .76 \\
\hline Metas contexto rendimiento (approach) & .74 & .70 \\
\hline Metas contexto rendimiento (avoidance) & .86 & .83 \\
\hline Autoeficacia & .82 & .78 \\
\hline Estrategias self-handicapping & .79 & .84 \\
\hline
\end{tabular}

Como se puede observar, los coeficientes alfa de Cronbach son aceptables, situándose entre .74 y .89, lo que evidencia un grado de consistencia interna razonablemente adecuado.

\subsection{ANÁLISIS FACTORIAL CONFIRMATORIO (CFA)}

La primera aproximación al estudio de la dimensionalidad de los cuestionarios se ha llevado a cabo a través de un análisis factorial exploratorio, realizando una rotación oblicua con normalización de Kaiser y estimación iterativa de las comunalidades, estableciendo el valor de delta (grado de oblicuidad entre factores) en -2.0. Se utilizó el método PAF, Principal Axis Factoring, para llevar a cabo este análisis de los ítems de las escalas seleccionadas. Al hilo de las evidencias encontradas, y comprobando que todos los ítems saturaban más en el factor que les correspondía, se decidió continuar hacia adelante con el estudio realizando un análisis factorial confirmatorio (CFA).

En este CFA, a diferencia del anterior, se parte de alguna hipótesis explícita acerca de la estructura de los factores que constituyen el modelo que se pretende probar (Brown, 2006). En nuestro caso, basándonos en los estudios previos de Midgley y sus colaboradores, consideramos que el conjunto de ítems (variables) de las diferentes subescalas del PALS son indicadores de 8 dimensiones latentes diferenciadas: metas aprendizaje, metas rendimiento (approach), metas rendimiento (avoidance), metas contexto aprendizaje, metas contexto rendimiento (approach), metas contexto rendimiento (avoidance), autoeficacia y estrategias self-handicapping. Consideramos que dichas dimensiones deben mostrar correlaciones positivas entre ellas, como suele ser el caso entre los constructos motivacionales.

A través del programa AMOS, se ha probado un modelo de medida con 8 factores de primer orden correlacionados entre sí. Cada uno de ellos está definido por cada una de las subescalas, de acuerdo con el modelo teórico.

En las tablas 4, 5 y 6 se recogen los resultados obtenidos: 
Tabla 4. Valor de las saturaciones de los ítems del PALS

\begin{tabular}{|c|c|c|c|c|c|c|c|c|}
\hline & MA & MRAP & MRAV & $\mathrm{MCA}$ & MCRAP & MCRAV & $\mathrm{AE}$ & $\mathrm{SH}$ \\
\hline Ítem 4 & .72 & .00 & .00 & .00 & .00 & .00 & .00 & .00 \\
\hline Ítem 9 & .82 & .00 & .00 & .00 & .00 & .00 & .00 & .00 \\
\hline Ítem 11 & .87 & .00 & .00 & .00 & .00 & .00 & .00 & .00 \\
\hline Ítem 13 & .68 & .00 & .00 & .00 & .00 & .00 & .00 & .00 \\
\hline Ítem 20 & .81 & .00 & .00 & .00 & .00 & .00 & .00 & .00 \\
\hline Ítem 3 & .00 & .73 & .00 & .00 & .00 & .00 & .00 & .00 \\
\hline Ítem 10 & .00 & .79 & .00 & .00 & .00 & .00 & .00 & .00 \\
\hline Ítem 14 & .00 & .76 & .00 & .00 & .00 & .00 & .00 & .00 \\
\hline Ítem 17 & .00 & .83 & .00 & .00 & .00 & .00 & .00 & .00 \\
\hline Ítem 19 & .00 & .84 & .00 & .00 & .00 & .00 & .00 & .00 \\
\hline Ítem 2 & .00 & .00 & .62 & .00 & .00 & .00 & .00 & .00 \\
\hline Ítem 12 & .00 & .00 & .68 & .00 & .00 & .00 & .00 & .00 \\
\hline Ítem 21 & .00 & .00 & .70 & .00 & .00 & .00 & .00 & .00 \\
\hline Ítem 23 & .00 & .00 & .76 & .00 & .00 & .00 & .00 & .00 \\
\hline Ítem 26 & .00 & .00 & .00 & .63 & .00 & .00 & .00 & .00 \\
\hline Ítem 28 & .00 & .00 & .00 & .49 & .00 & .00 & .00 & .00 \\
\hline Ítem 30 & .00 & .00 & .00 & .64 & .00 & .00 & .00 & .00 \\
\hline Ítem 33 & .00 & .00 & .00 & .53 & .00 & .00 & .00 & .00 \\
\hline Ítem 35 & .00 & .00 & .00 & .79 & .00 & .00 & .00 & .00 \\
\hline Ítem 37 & .00 & .00 & .00 & .47 & .00 & .00 & .00 & .00 \\
\hline Ítem 29 & .00 & .00 & .00 & .00 & .75 & .00 & .00 & .00 \\
\hline Ítem 31 & .00 & .00 & .00 & .00 & .65 & .00 & .00 & .00 \\
\hline Ítem 38 & .00 & .00 & .00 & .00 & .69 & .00 & .00 & .00 \\
\hline Ítem 27 & .00 & .00 & .00 & .00 & .00 & .78 & .00 & .00 \\
\hline Ítem 32 & .00 & .00 & .00 & .00 & .00 & .71 & .00 & .00 \\
\hline Ítem 34 & .00 & .00 & .00 & .00 & .00 & .72 & .00 & .00 \\
\hline Ítem 36 & .00 & .00 & .00 & .00 & .00 & .77 & .00 & .00 \\
\hline Ítem 39 & .00 & .00 & .00 & .00 & .00 & .72 & .00 & .00 \\
\hline Ítem 1 & .00 & .00 & .00 & .00 & .00 & .00 & .62 & .00 \\
\hline Ítem 5 & .00 & .00 & .00 & .00 & .00 & .00 & .63 & .00 \\
\hline Ítem 22 & .00 & .00 & .00 & .00 & .00 & .00 & .69 & .00 \\
\hline Ítem 24 & .00 & .00 & .00 & .00 & .00 & .00 & .80 & .00 \\
\hline Ítem 25 & .00 & .00 & .00 & .00 & .00 & .00 & .73 & .00 \\
\hline Ítem 6 & .00 & .00 & .00 & .00 & .00 & .00 & .00 & .61 \\
\hline Ítem 7 & .00 & .00 & .00 & .00 & .00 & .00 & .00 & .67 \\
\hline Ítem 8 & .00 & .00 & .00 & .00 & .00 & .00 & .00 & .60 \\
\hline Ítem 15 & .00 & .00 & .00 & .00 & .00 & .00 & .00 & .61 \\
\hline Ítem 16 & .00 & .00 & .00 & .00 & .00 & .00 & .00 & .66 \\
\hline Ítem 18 & .00 & .00 & .00 & .00 & .00 & .00 & .00 & .60 \\
\hline
\end{tabular}

MA= Metas aprendizaje; MRAP= Metas rendimiento (approach); MRAV= Metas rendimiento (avoidance); MCA= Metas contexto aprendizaje; MCRAP= Metas contexto rendimiento $($ approach $) ; \mathbf{M C R A V}=$ Metas contexto rendimiento (avoidance); $\mathbf{A E}=$ Autoeficacia; $\mathbf{S H}=$ Estrategias self-handicapping. 
Estudios Pedagógicos XLII, N 3: 391-412, 2016

ESTUDIO INICIAL DE LAS PROPIEDADES PSICOMÉTRICAS DE LAS ESCALAS MOTIVACIONALES DEL PALS (PATTERNS OF ADAPTIVE LEARNING SCALES) CENTRADAS EN EL ALUMNO

Tabla 5. Índices de bondad de ajuste del modelo de primer orden comparados con los del modelo independiente

\begin{tabular}{|c|c|c|}
\hline & $\begin{array}{c}\text { MODELO } \\
\text { DE PRIMER ORDEN }\end{array}$ & $\begin{array}{c}\text { MODELO INDEPENDIENTE } \\
\text { (NULO) }\end{array}$ \\
\hline CMIN & 1306.984 & 7530.117 \\
\hline $\mathrm{GL}$ & 674 & 780 \\
\hline $\mathrm{p}$ & .000 & .000 \\
\hline $\mathrm{CMIN} / \mathrm{GL}$ & 1.939 & 9.654 \\
\hline $\mathrm{CFI}$ & .906 & .000 \\
\hline RMSEA & .050 & .152 \\
\hline
\end{tabular}

Tabla 6. Matriz de correlación entre los factores motivacionales

\begin{tabular}{|l|c|c|c|c|c|c|c|c|}
\hline & MA & MRAP & MRAV & MCA & MCRAP & MCRAV & AE & SH \\
\hline MA & - & & & & & & & \\
\hline MRAP & .14 & - & & & & & & \\
\hline MCA & .81 & .07 & .09 & - & & & & \\
\hline MCRAP & .17 & .46 & .58 & .23 & - & & & \\
\hline MCRAV & .04 & .87 & .94 & .02 & .62 & - & & \\
\hline AE & .51 & .13 & .11 & .50 & .24 & .06 & - & \\
\hline SH & -.40 & .33 & .25 & -.34 & .14 & .34 & -.25 & - \\
\hline
\end{tabular}

Como puede verse en la Tabla 5, el valor de Chi cuadrado es de 1306.984 con 674 grados de libertad $(\mathrm{p}=.000)$. Sin embargo, este estadístico está muy afectado por el tamaño de la muestra, por lo que no suele emplearse como buen indicador de la bondad del modelo (Jöreskog, 1969). El valor de Chi cuadrado relativo es de 1.939, lo que representa un ajuste razonable del modelo a los datos. El CFI alcanza un valor moderadamente alto (.906). Finalmente, el RMSEA tiene un valor de .050, lo que según Browne y Cudeck (1993) indica un razonable error de especificación del modelo.

Otro modo comúnmente utilizado para valorar estos índices es compararlos con aquellos correspondientes al modelo nulo (en nuestro caso, el modelo independiente), definido como aquel en el que se supone que las variables no están relacionadas entre sí. El objeto del modelo nulo es proporcionar una referencia con la que poder comparar otros modelos, en este caso, el modelo de 8 factores. Un buen ajuste del modelo nulo a los datos supondría que el intento de explicar una cierta estructura interna de los mismos carece 
de sentido. Por el contrario, si el modelo nulo no se ajusta a los datos, las correlaciones entre las distintas variables observadas son estadísticamente significativas y, por tanto, tiene sentido el intentar explicar las asociaciones entre las variables medidas a través de un conjunto de parámetros, menor en número que el de covarianzas intervariables. Como puede observarse, los valores correspondientes al modelo independiente (modelo nulo) son claramente diferentes a los del modelo propuesto. Por ejemplo, la discrepancia entre el modelo y los datos es casi seis veces mayor en aquel, y cuando este valor se divide por sus grados de libertad sigue siendo casi cinco veces mayor. Del mismo modo, el RMSEA es más del triple que el obtenido para el modelo original. Estos resultados nos indican que es muy razonable mantener, frente al modelo independiente, una estructura en las que las variables motivacionales medidas están explicadas por los ocho factores latentes relacionados entre sí.

Los autores del PALS han realizado diferentes análisis confirmatorios, con objeto de examinar la estructura subyacente al conjunto de variables (ítems) que hacen referencia a metas personales y contextuales (Anderman et al., 2003; Midgley, 2002; Midgley et al., 2000; Wolters, 2004). En el primer caso, y empleando el programa LISREL VIII, los índices de ajuste que presentan $(\mathrm{GFI}=.97, \mathrm{AGFI}=.95)$ confirman el modelo compuesto por tres factores (metas aprendizaje, metas rendimiento approach y metas rendimiento avoidance). Respecto a las metas contextuales, también encuentran evidencia empírica $(\mathrm{GFI}=.96, \mathrm{AGFI}=.94)$ que confirma la estructura subyacente (metas contexto aprendizaje, metas contexto rendimiento approach y metas contexto rendimiento avoidance).

Por otro lado, en la Tabla 6 se recogen las correlaciones entre los distintos factores motivacionales. Como se puede observar, el patrón de relaciones es muy similar al obtenido al correlacionar las puntuaciones de los participantes en cada subescala, aunque las magnitudes de los valores es ligeramente diferente. Por ejemplo, el factor metas de aprendizaje presenta una correlación de .51 con el factor autoeficacia, mientras que con el factor estrategias self-handicapping la correlación es negativa (-.40).

\section{DISCUSIÓN}

Como se evidencia, el comportamiento psicométrico de los ítems de las escalas utilizadas es bastante aceptable, ya que cada ítem, con las matizaciones puestas de manifiesto, está significativamente correlacionado con aquellos otros diseñados para medir su misma dimensión. Sin embargo, la baja correlación de alguno de ellos con su respectiva escala parece hacer aconsejable una revisión en la formulación y adaptación de los mismos. En concreto, respecto al ítem 28, hemos de destacar que su falta de correlación puede deberse a que su formulación es difícil de comprender para un alumno de 13-14 años, ya que se le pregunta si en su clase se valora el grado de progreso personal, aspecto que implica un alto grado de conciencia que en esas edades consideramos difícil de alcanzar de un modo realista y objetivo. En relación con los otros dos ítems (33 y 37), la introducción de una negación en sus correspondientes enunciados puede haber causado algún problema de comprensión a los alumnos durante la aplicación de las pruebas, lo que nos obliga a plantearnos una revisión de los mismos.

En cuanto a la fiabilidad, los coeficientes alfa de Cronbach son bastante altos (entre .74 y .89), lo que muestra un elevado grado de consistencia interna. Asimismo, comparando 
nuestros datos con los que aparecen en el Manual del instrumento original, se observa que nuestros valores son iguales o superiores, menos en la de estrategias self-handicapping, el cual es menor (aunque no es un valor significativo).

En cuanto al análisis factorial confirmatorio realizado, observamos que nuestra versión se ajusta, con las limitaciones puestas de manifiesto, al modelo propuesto. En concreto, el CFA confirma el modelo de ocho factores propuesto por Midgley y sus colaboradores. Sin embargo, se requiere cautela, ya que el grado de ajuste del modelo no es tan alto como sería deseable. En este sentido, hemos observado que los dos componentes (approachavoidance) diferenciados en las metas de rendimiento no aparecen en nuestro estudio claramente diferenciados (cosa que sucede también con las metas contexto rendimiento). Como posible explicación se podría decir, tal y como evidenciaron Elliot y Church (1997), que en las dos subdivisiones de las metas de rendimiento está presente, como disposición que dirige y organiza la conducta del sujeto, el miedo al fracaso. Por otra parte, atendiendo al contenido de los ítems de las dos dimensiones, la mayoría de ellos hace referencia a la comparación social, lo que puede explicar la conexión entre ambas. Asimismo, hay que tener en cuenta que cuando los investigadores han tratado de operativizar las metas de rendimiento han tendido casi exclusivamente a proponer ítems que las definen poniendo el foco en la demostración de competencia (en relación a otros). Sin embargo, cuando a los estudiantes se les permite describir sus metas con sus propias palabras, rara vez mencionan espontáneamente este tipo de metas. Los estudiantes pueden señalar que aspiran a pasar un examen y conseguir una buena nota, pero no mencionan el demostrar capacidad o parecer bueno en relación con sus compañeros como posibles motivos de su conducta.

Ello nos da a entender, en consonancia con lo que señala Brophy (2005), que los instrumentos comúnmente empleados por los investigadores para evaluar estas metas suelen mostrar una alta consistencia interna (metodológicamente hablando), pero no responden claramente a la realidad, pues hay fuerte evidencia de que los estudiantes no piensan esas metas tal como las definen los investigadores. Es decir, no tienden a plantearse metas de rendimiento que incluyen elementos en relación a competición y comparación social (lo que puede ser tal vez especialmente más cierto en nuestro contexto que en el americano). Por ello, Brophy señala que se debería reformular el concepto de metas de rendimiento, distinguiendo diferentes subtipos (tal como proponen Grant y Dweck, 2003): obtener resultados positivos (ir bien en el curso, conseguir buenas notas), validar la propia capacidad (demostrar o confirmar que se tiene capacidad para realizar el trabajo escolar) y metas normativas que incluyen la comparación social (confirmar que se es más inteligente o que uno va mejor que otros en la clase). Admitir estas distinciones ayudaría a clarificar la controversia y confusión que existe sobre las metas de rendimiento tal como indican numerosos estudios empíricos, y entre ellos, el nuestro.

En conclusión, y en coherencia con los estudios previos realizados en esta área (por ejemplo, Anderman et al., 2003; Cheng y Lam, 2013; Ross, Blackburn y Forbes, 2005), los resultados evidencian que nuestra versión de las escalas del PALS utilizadas posee, con algunas matizaciones, unas propiedades psicométricas relativamente adecuadas como para poder aplicarse en la práctica educativa y en la investigación sobre metas académicas en alumnos de educación secundaria. Sin embargo, se requiere seguir realizando estudios de validación relativos a la distinción entre las dimensiones approach y avoidance y acerca de la diferenciación entre las metas personales y las referidas al contexto. 


\section{LIMITACIONES Y FUTURAS DIRECCIONES}

Para explicar algunas de las inconsistencias halladas, también se podría añadir que, aunque nuestra traducción y adaptación se verificó con profesores bilingües y las escalas se sometieron a un análisis de validación de contenido con una muestra piloto, algunos de los ítems pueden no haber resultado claros para los estudiantes (por ejemplo, algunos de los formulados en sentido inverso), provocando una mala comprensión de los mismos, que podría haber afectado a las escalas con menores índices de consistencia interna. Por otro lado, de cara a lograr una mayor garantía de validez, creemos necesario complementar nuestro estudio utilizando nuevas muestras, nuevos contextos socioculturales (diferentes al norteamericano donde se validó el instrumento), nuevos modelos y teniendo en cuenta diferentes condiciones contextuales del proceso de enseñanza (Tourón y Santiago, 2015). Otro aspecto importante es que si bien la escala se amplió a 7 puntos, no deja de ser strictu sensu una escala ordinal, por lo que sería interesante comprobar el análisis de la estructura empleando un análisis factorial ordinal que se adapte mejor a la métrica de las variables (cfr. Tourón, Lizasoain y Sobrino, 2014), lo que abordaremos en ulteriores estudios.

A pesar del fallecimiento en la década pasada de Carol Midgley, precursora y fundadora de este instrumento, su empleo se ha generalizado extensamente en la literatura sobre metas académicas (Achievement goal theory) y en otros campos. De hecho, podemos afirmar que al día de hoy no ha perdido su protagonismo, siendo en la actualidad un gran número de estudios los que lo utilizan como marco de referencia. Gracias a él se han podido determinar y diferenciar de una manera más precisa los aspectos motivacionales relacionados con el logro académico, lo que le ha convertido en una herramienta con una adecuada fiabilidad y validez que capacita para evaluar los aspectos motivaciones implicados en el aprendizaje autorregulado.

\section{REFERENCIAS BIBLIOGRÁFICAS}

Ames, C. (1992). Classrooms: Goals, structures, and student motivation. Journal of Educational Psychology, 84, 261-271. doi:10.1037//0022-0663.84.3.261

Anderman, E. M., \& Midgley, C. (1997). Changes in achievement goal orientations, perceived academic competence, and grades across the transition to middle-level schools. Contemporary Educational Psychology, 22, 269-298. doi:10.1006/ceps.1996.0926

Anderman, E. M., \& Wolters, C. (2006). Goals, values, and affects: influences on student motivation. En P. Alexander \& P. Winne (Eds.), Handbook of Educational Psychology (pp. 369-389). New York: Simon y Schuster/Macmillan. doi:10.4324/9780203874790.ch17

Anderman, E. M., Urdan, T., \& Roeser, R. (2003). The Patterns of Adaptive learning Survey: History, development and psychometric properties. Indicators of Positive Development Conference, Washington.

Anderman, L. H., \& Anderman, E. M. (1999). Social predictors of change in student's achievement goal orientation. Contemporary Educational Psychology, 23, 21-37.

Arbuckle, J. L. (2010). IBM SPSS Amos 19 User's Guide. Chicago, IL: SPSS.

Atkinson J. W. (1957). Motivational determinants of risk-taking behavior. Psychological Review, 64, 359-372.

Barron, K. E., \& Harackiewicz, J. M. (2003). Revisiting the benefits of performance-approach goals in the college classroom: Exploring the role of goals in advanced college courses. International Journal of Educational Research, 39, 357-374. doi:10.1016/j.ijer.2004.06.004 
Estudios Pedagógicos XLII, N 3: 391-412, 2016

ESTUDIO INICIAL DE LAS PROPIEDADES PSICOMÉTRICAS DE LAS ESCALAS MOTIVACIONALES DEL PALS

(PATTERNS OF ADAPTIVE LEARNING SCALES) CENTRADAS EN EL ALUMNO

Blanco, L.J. (2012). Influencias del dominio afectivo en la enseñanza y el aprendizaje de las matemáticas. En N. Planas (Coord.), Teoría, crítica y práctica de la educación matemática (pp. 171-185). Barcelona: Graó.

Brophy, J. (2005). Achievement goal researchers should move on from performance goals. Educational Psychologist, 40(3), 167-176.

Brown, T. A. (2006). Confirmatory factor analysis for applied research. New York: Guilford Press.

Browne, M. W., \& Cudeck, R. (1993). Alternative ways of assessing model fit. En K. A. Bollen \& J. S. Long (Eds.), Testing structural equation models (pp. 136-162). Newbury Park, CA: Sage. doi:10.1177/0049124192021002005

Butler, D. L., Schnellert, L., \& Cartier, S. C. (2013). Layers of self- and co-regulation: Teachers' co-regulating learning and practice to foster students' self-regulated learning through reading. Education Research International. doi:10.1155/2013/845694

Cheng, R. W., \& Lam, S. (2013). The interaction between social goals and self-construal on achievement motivation. Contemporary Educational Psychology, 38, 136-148. doi:10.1016/j. cedpsych.2013.01.001

Darnon, C., Harackiewicz, J. M., Butera, F., Mugny, G., \& Quiamzade, A. (2007). Performanceapproach and performance avoidance goals: When uncertainty makes a difference. Personality and Social Psychology Bulletin, 33, 813-827.

Daura, F. (2011). Las estrategias docentes al servicio del desarrollo del aprendizaje autorregulado. Estudios pedagógicos, 37(2), 77-88.

De la Fuente, J. (2004). Perspectivas recientes en el estudio de la motivación: la teoría de la orientación de meta. Revista Electrónica de Investigación Psicoeducativa, 2, 35-62.

Dignath, C., \& Büttner, G. (2008). Components of fostering self-regulated learning among students. A meta-analysis on intervention studies at primary and secondary school level. Metacognition and Learning, 3, 231-264. doi:10.1007/s11409-008-9029-x

Dweck, C. S. (1986). Motivational processes affecting learning. American Psychologist, 41, 10401048. doi:10.1037//0003-066X.41.10.1040

Elliot, A. J., \& Church, M. A. (1997). A Hierarchical model of approach and avoidance achievement Motivation. Journal of Personality and Social Psychology, 72, 218-232. doi:10.1037/00223514.72.1.218

Elliot, A. J., \& McGregor, H. (2001). A 2 x 2 achievement goal framework. Journal of Personality and Social Psychology, 80, 501-519.

Elliot, A. J., \& Moller, C. (2003) Performance-approach goals: good or bad forms of regulation? International Journal of Educational Research, 39, 339-356. doi:10.1016/j.ijer.2004.06.003

González-Cabanach, R., Valle, A. Rodríguez, S., García, M., \& Mendiri, P. (2007). Programa de intervención para mejorar la gestión de los recursos motivacionales en estudiantes universitarios. Revista Española de Pedagogía, 237, 237-256.

González-Torres, M. C. (1997). La motivación académica. Sus determinantes y pautas de intervención. Pamplona: EUNSA.

González-Torres, M. C., \& Torrano, F. (2008). Methods and instruments for measuring self-regulated learning. En A. Valle y J. C. Núñez (Eds.), Handbook of instructional resources and their applications in the classroom (pp. 201-219). Hauppauge, NY: Nova Science.

González-Torres, M. C., \& Torrano, F. (2013). Perfiles de motivación y rendimiento académico en matemáticas en estudiantes de educación secundaria: Utilidad del Patterns of Adaptive Learning Scales (PALS). En V. Mellado, L.J. Blanco, A.B. Borrachero y J.A. Cárdenas (Eds.), Las Emociones en la Enseñanza y el Aprendizaje de las Ciencias Experimentales y las Matemáticas (pp. 177-215). Badajoz: DEPROFE.

Grant, H., \& Dweck, C. S. (2003). Clarifying achievement goals and their impact. Journal of Personality and Social Psychology, 85, 541-553.

Harackiewicz, J. M., Barron, K. E., Pintrich, P. R., Elliot, A. E., \& Thrash, T. M. (2002). Revision of 
achievement goal theory: Necessary and illuminating. Journal of Educational Psychology, 94, 638-645. doi:10.1037/0022-0663.94.3.638

Harackiewicz, J. M., Durik, A.M., Barron, K.E., Linnenbrink, L., \& Tauer, J. (2008). The role of achievement goals in the development of interest: Reciprocal relations between achievement goals, interest, and performance. Journal of Educational Psychology, 100, 105-122. doi:10.1037/0022-0663.100.1.105

Hershkovitz, A., Baker, R.S., Gobert, J., Wixon, M., \& Sao Pedro, M. (2013). Discovery with models: A case study on carelessness in computer-based science inquiry. American Behavioral Scientist, $57,1479-1498$.

Hoffman, R. L., Hudak-Rosander, C., Datta, J., Morris, J. B., \& Kelz, R. R. (2014). Goal orientation in surgical residents: a study of the motivation behind learning. Journal of Surgical Research, 190, 451-460. doi:10.1016/j.jss.2014.01.005

Hulleman, C.S., Durik, A.M., Schweigert, S.A., \& Harackiewicz, J.M. (2008). Task values, achievement goals, and interest. Journal of Educational Psychology, 100, 398-416.

Jöreskog, K. G. (1969). A General approach to confirmatory maximum likehood factor analysis. Psychometrika, 34, 183-202.

Kaplan, A., \& Maehr, M. L. (2007). The contributions and prospects of goal orientation theory. Educational Psychology Review, 19, 141-184. doi:10.1007/s10648-006-9012-5

Kaplan, A., \& Midgley, C. (1997). The effect of achievement goals: Does level of perceived academic competence make a difference? Contemporary Educational Psychology, 22, 415-435. doi:10.1006/ceps.1997.0943

Linnenbrink, E. A. (2005). The dilemma of performance-approach goals: The use of multiple goal contexts to promote students' motivation and learning. Journal of Educational Psychology, 97, 197-213.

Linnenbrink, E. A., \& Pintrich P. R. (2003). The role of self-efficacy beliefs in student engagement and learning in the classroom. Reading Writing Quarterly, 19, 119-137.

Madjar, N., Bachner, Y. G., \& Kushnir, T. (2011). Can achievement goal theory provide a useful motivational perspective for explaining psychosocial attributes of medical students? BMC Medical Education, 12(4). doi:10.1186/1472-6920-12-4

Maehr, M. L., \& Zusho, A. (2009). Achievement goal theory: The past, present, and future. En K. R. Wentzel y A. Wigfield (Eds.), Handbook of motivation in school (pp. 77-104). New York: Taylor \& Francis.

Meece, J. L., Anderman, E. M., \& Anderman, L. H. (2006). Classroom goal structures, student motivation, and academic achievement. Annual Review of Psychology, 57, (pp. 487-503). Chippewa Fall, WI: Annual Reviews. doi:10.1146/annurev.psych.56.091103.070258

Midgley, C. (Ed.). (2002). Goals, goal structure and pattern of adaptive learning. Mahwah, NJ: Lawrence Erlbaum.

Midgley, C., Kaplan, A., \& Middleton, M. (2001). Performance-approach goals: Good for what, for whom, under what circumstances, and at what cost? Journal of Educational Psychology, 9, 7786. doi:10.1037/0022-0663.93.1.77

Midgley, C., Maehr, M. L., Hruda, L. Z., Anderman, E., Anderman, L., Freeman, K. E.,... Gheen, M. (2000). Manual for the Patterns of Adaptive Learning Scales (PALS). Ann Arbor, MI: University of Michigan. Recuperado de: http://www.umich.edu/ pals/pals/PALS\%202000_V13Word97. pdf

Midgley, C., \& Urdan, T. (2001). Academic self-handicapping and achievement goals: A further examination. Contemporary Educational Psychology, 26, 61-75. doi:10.1006/ceps.2000.1041

Muis, K. R., \& Winne, P. H. (2012). Assessing the psychometric properties of the achievement goals questionnaire Across Task Contexts. Canadian Journal of Education, 35, 232-248.

Negru, O., \& Damian, L. (2010). Personal and classroom promoted achievement goals: Interdependence between students and teachers. Cognition, Brain, Behavior, 14, 81-99. 
Estudios Pedagógicos XLII, N 3: 391-412, 2016

ESTUDIO INICIAL DE LAS PROPIEDADES PSICOMÉTRICAS DE LAS ESCALAS MOTIVACIONALES DEL PALS

(PATTERNS OF ADAPTIVE LEARNING SCALES) CENTRADAS EN EL ALUMNO

Niemivirta, M. (1998). Individual differences in motivational and cognitive factors affecting selfregulated learning: A pattern-oriented approach. In P. Nenniger, R. S. Jager, \& M. Wosnitza (Eds.), Advances in Motivation (pp. 32-42). Landau: Verlag Empirische Padagogik.

Norusis, M. (2011). IBM SPSS Statistics 19: Guide to data analysis. Chicago, IL: SPSS.

Patrick, H., Anderman, L. H., \& Ryan, A. M. (2002). Social motivation and the classroom social environment. En C. Midgley (Ed.), Goals, Goal Structure and Pattern of Adaptative Learning (pp. 85-108). Mahwah, NJ: Lawrence Erlbaum.

Patrick, H., Ryan, A. M., \& Kaplan, A. (2007). Early adolescents' perceptions of the classroom social environment, motivational beliefs, and engagement. Journal of Educational Psychology, 99, 83-98.

Pintrich, P. R., Smith, D., García, T., \& McKeachie, W. J. (1991). A Manual for the Use of the Motivated Strategies for Learning Questionnaire (MSLQ). Ann Arbor, MI: University of Michigan.

Putwain, D. (2013). Conceptualizing and measuring academic motivation. Assessment and Development Matters, 5, 26-29.

Roces, C., Tourón, J., \& González-Torres, M. C. (1995). Validación preliminar del CEAM II. Psicológica, 16, 347-366.

Rosario, P., Núñez, J. C., Trigo, L., Guimaraes, C., Fernández, E., Cerezo, R.,... Fuentes, S. (2015). Transcultural analysis of the effectiveness of a program to promote self-regulated learning in Mozambique, Chile, Portugal, and Spain. Higher Education Research \& Development, 34, 173187. doi:10.1080/07294360.2014.935932

Ross, M. E., Blackburn, M., \& Forbes, S. (2005). Reliability generalization of the Patterns of Adaptive Learning Survey goal orientation Scales. Educational and Psychological Measurement, 65, 451464. doi: $10.1177 / 0013164404272496$

Sarabia, A., \& Iriarte, C. (2011). El aprendizaje de las matemáticas: ¿qué actitudes, creencias y emociones despierta esta materia en los alumnos? Pamplona: EUNSA.

Schunk, D. H., \& Zimmerman, B. J. (2007). Motivation and self-regulated learning: Theory, research, and applications. Mahwah, NJ: Lawrence Erlbaum.

Senko, C., Hulleman, C. S., \& Harackiewicz, J. M. (2011). Achievement goal theory at the crossroads: Old controversies, current challenges, and new directions. Educational Psychologist, 46, 26-47.

Skaalvik, E. M. (1997). Self-enhancing and self-defeating ego orientation: Relations with task and avoidance orientation, achievement, self-perceptions, and anxiety. Journal of Educational Psychology, 89, 71-81

Smith, L., \& Sinclair, K. E. (2005) Empirical evidence for multiple goals: A gender-based, senior high school student perspective. Australian Journal of Educational \& Developmental Psychology, 5, 55-70.

Strobel, K. (2010). Practices that promote Middle School students'motivation and achievement. John W. Gardner Center for Youth and Their Communities Stanford School of Education.

Torrano, F., \& González-Torres, M. C. (2004). El aprendizaje autorregulado: presente y futuro de la investigación. Revista Electrónica de Investigación Psicoeducativa, 2, 1-34.

Tourón, J., Lizasoain, L., \& Sobrino, A. (2014). Análisis del impacto del feedback en las prácticas docentes de los profesores españoles del estudio TALIS 2013. Informe español: TALIS 2013. Estudio Internacional de la enseñanza y el aprendizaje. Análisis Secundario (pp. 229-246). Madrid: Instituto Nacional de Evaluación Educativa.

Tourón, J., y Santiago, R. (2015). El modelo Flipped Learning y el desarrollo del talento en la escuela. Revista de Educación, 368, 196-231. doi:10.4438/1988-592X-0034-8082-RE

Turner, J. C., Midgley, C., Meyer, D. K., Gheen, M., Anderman, E. M., Kang, Y., \& Patrick, H. (2002). The classroom environment and students' reports of avoidance strategies in mathematics: A multimethod study. Journal of Educational Psychology, 94, 88-106.

Urdan, T. (2004). Predictors of academic self-handicapping and achievement: Examining achievement 
goals, classroom goal structures, and culture. Journal of Educational Psychology, 96, 251-264. doi:10.1037/0022-0663.96.2.251

Urdan, T., \& Schoenfelder, E. (2006). Classroom effects on student motivation: Goal structures, social relationships, and competence beliefs. Journal of School Psychology 44, 331-349. doi:10.1016/j. jsp.2006.04.003

Valle, A., Rodríguez, S., Cabanach, R. G., Núñez, J. C., González-Pienda, J. A., \& Rosario, P. (2009). Metas académicas: perspectiva histórica y conceptual e implicaciones educativas. Electronic Journal of Research in Educational Psychology, 7, 1073-1106.

Weinstein, C. E., Schulte, A. C., \& Palmer, D. R. (1987). LASSI: Learning and Study Strategies Inventory. Clearwater, FL: $\mathrm{H}$ y $\mathrm{H}$ Publishing.

Wentzel, K. R. (2003). School adjustment. En W. M. Reynolds \& G. E. Miller (Eds.), Handbook of Psychology: Educational Psychology (Vol. 7, pp. 235-258). Hoboken, NJ: Wiley.

Winne, P. H. (en prensa). Self-regulated learning. En J. D. Wright (Ed.), International encyclopedia of social \& behavioral sciences (2nd ed.). Elsevier: Oxford, UK.

Wigfield, A., \& Cambria, J. (2010). Students' achievement values, goal orientations, and interest: Definitions, development, and relations to achievement outcomes. Developmental Review, 30, $1-35$.

Wolters C. (2004). Advancing achievement goal theory: using goal structures and goal orientations to predict students' motivation, cognition, and achievement. Journal of Educational Psychologist, 96, 236-250.

Zimmerman, B. J. (2008). Investigating self-regulation and Motivation: Historical Background, methodological developments, and future prospects. American Educational Research Journal, 45, 166-183.

Zimmerman, B. J., \& Schunk, D. H. (2011). Handbook of self-regulation of learning and performance. New York: Routledge. 


\section{CUESTIONARIO DE MOTIVACIÓN ACADÉMICA}

\section{METAS, CREENCIAS DE AUTOEFICACIA Y ESTRATEGIAS MOTIVACIONALES EGODENFENSIVAS}

\section{Basado en el Patterns of Adaptive Learning Scales (PALS) University of Michigan (2000)}

Traducción y adaptación: Torrano, F. y González-Torres, M. C. Departamento de Educación, Universidad de Navarra

El profesor que aplica este cuestionario está participando en una investigación sobre la enseñanza y el aprendizaje en la etapa de educación secundaria obligatoria. Nos gustaría que colaborases en este estudio, respondiendo a unas preguntas referidas a tu motivación en la asignatura de matemáticas de este curso. La participación es voluntaria y no influye en ninguna nota.

Queremos que recuerdes que no hay respuestas correctas o incorrectas. Esto no es un examen. Queremos que respondas con la mayor sinceridad y precisión posible, reflejando tu propia actitud y tu motivación en los estudios.

Por favor, antes de comenzar cumplimente los datos que aparecen en la parte superior de la hoja de respuesta. No escribas nada en este cuadernillo.

Gracias por tu participación y tu colaboración. 
A continuación se te hacen unas preguntas para conocer algunos aspectos de ti mismo como estudiante y de tu clase de matemáticas. Es importante que recuerdes que no existen respuestas correctas o incorrectas; intenta solamente contestar, reflejando de la manera más exacta tu propia situación. Usa la escala que te ofrecemos a continuación para contestar a las preguntas. Si una afirmación describe tu situación personal perfectamente, debes darle una puntuación de 7; si no la describe en absoluto, dale la puntuación 1. Si la afirmación se ajusta en tu caso sólo en parte, o sólo en algunas ocasiones, encuentra en número entre el 1 y el 7 que mejor te describa. Contesta a todas las preguntas en la hoja de respuestas, no escribas nada en este cuadernillo.

\begin{tabular}{|ccccccc|}
\hline 1 & 2 & 3 & 4 & 5 & 6 & 7 \\
$\begin{array}{c}\text { No } \\
\text { Nunca }\end{array}$ & & $\begin{array}{c}\text { Más bien no } \\
\text { Alguna vez }\end{array}$ & & $\begin{array}{c}\text { Más bien sí } \\
\text { Con bastante } \\
\text { frecuencia }\end{array}$ & & $\begin{array}{c}\text { Sí } \\
\text { Siempre }\end{array}$ \\
\hline
\end{tabular}

1. Estoy seguro de que puedo dominar los conocimientos y las habilidades que se van a enseñar este año en clase de matemáticas.

2. Para mí es importante no parecer tonto en clase de matemáticas.

3. Para mí es importante que los demás compañeros piensen que se me dan bien las tareas de matemáticas.

4. Para mí es importante aprender muchos conceptos nuevos este año en matemáticas.

5. Estoy seguro de que puedo entender cómo hacer las tareas de matemáticas más difíciles.

6. Algunos estudiantes pierden el tiempo la noche anterior al examen de matemáticas de forma que, si sus resultados no son buenos, pueden decir que esa es la causa. ¿En qué medida tú también haces esto?

7. Algunos estudiantes intencionadamente se implican en muchas actividades. Así, si sus resultados en matemáticas no son buenos, pueden decir que es debido a que estaban comprometidos con otras cosas. ¿En qué medida tú también haces esto?

8. Algunos estudiantes buscan excusas para no estudiar (no sentirse bien, tener que ayudar a sus padres, tener al cuidado un hermano o hermana, etc.). Así, si sus resultados en matemáticas no son buenos, pueden decir que esa es la causa. ¿En qué medida tú también haces esto?

9. Una de mis metas en clase de matemáticas es aprender lo máximo que pueda.

10. Una de mis metas es que los demás vean que se me dan bien las tareas de matemáticas.

11. Una de mis metas este año en matemáticas es adquirir gran cantidad de conocimientos y habilidades nuevas.

12. Una de mis metas es evitar que los otros piensen que no soy inteligente en clase de matemáticas.

13. Para mí es importante entender a fondo las tareas de matemáticas.

14. Una de mis metas es que los demás vean que las tareas de matemáticas me resultan fáciles.

15. Algunos estudiantes permiten que sus amigos les impidan prestar atención durante la clase o la realización de las tareas de matemáticas. Así, si sus resultados no son 
buenos, pueden decir que sus amigos les impidieron trabajar. ¿En qué medida tú también haces esto?

16. Algunos estudiantes intencionadamente no se esfuerzan en clase de matemáticas de forma que, si sus resultados no son buenos, pueden decir que es debido a que no se esforzaron. ¿En qué medida tú también haces esto?

17. Una de mis metas es parecer inteligente, en comparación con los demás compañeros de mi clase de matemáticas.

18. Algunos estudiantes dejan sus tareas de matemáticas para el último momento de forma que, si sus resultados no son buenos, pueden decir que ese retraso fue la razón. ¿En qué medida tú también haces esto?

19. Para mí es importante parecer inteligente, en comparación con los demás compañeros de mi clase de matemáticas.

20. Para mí es importante mejorar mis conocimientos y mis habilidades este año en matemáticas.

21. Para mí es importante que el profesor no piense que sé menos que los demás compañeros de mi clase de matemáticas.

22. Puedo hacer prácticamente todas las tareas de matemáticas si no me rindo.

23. Una de mis metas en clase de matemáticas es evitar parecer que tengo dificultades a la hora de realizar las tareas.

24. Incluso si la tarea de matemáticas es difícil, puedo aprenderla.

25. Puedo hacer hasta la tarea de matemáticas más difícil si lo intento.

26. En clase de matemáticas, esforzarse es muy importante.

27. En clase de matemáticas, mostrar a los otros que no eres malo en las tareas es muy importante.

28. En clase de matemáticas, el grado de progreso personal es realmente muy importante.

29. En clase de matemáticas, conseguir buenas notas es el principal objetivo.

30. En clase de matemáticas, el objetivo principal es comprender bien el material de aprendizaje.

31. En clase de matemáticas, dar las respuestas correctas es muy importante.

32. En clase de matemáticas, es importante no cometer errores a la vista de todo el mundo.

33. En clase de matemáticas, es importante entender las tareas, no simplemente memorizarlas.

34. En clase de matemáticas, es importante no tener peores resultados que otros estudiantes.

35. En clase de matemáticas, aprender ideas y conceptos nuevos es muy importante.

36. En clase de matemáticas, es importante no parecer tonto.

37. En clase de matemáticas, no importa cometer errores, siempre que estés aprendiendo.

38. En clase de matemáticas, es importante conseguir notas altas en los exámenes.

39. En clase de matemáticas, uno de los principales objetivos es evitar parecer que no puedes hacer las tareas. 